\title{
A NOTE ON UNITS OF ALGEBRAIC NUMBER FIELDS
}

\author{
TOMIO KUBOTA
}

We shall prove in the present note a theorem on units of algebraic number fields, applying one of the strongest formulations, be Hasse [3], of Grunwald's existence theorem.

Theorem. Let $k$ be an algebraic number field, $l$ a prime number, $E_{k}$ the group of units of $k$ and $H$ a subgroup of $E_{k}$ containing all l-th powers of elements of $E_{k}$. Assume that, for every $\eta \in H, k(\sqrt[l]{\eta})$ is always ramified over $k$ whenever $k$ contains an $l$-th root $\zeta_{l}(\neq 1)$ of unity. Then there are infinitely many cyclic extentions $K / k$ of degree $l$ with following properties:

a) $N_{K / k} E_{K}=H$, where $E_{K}$ is the group of units of $K$.

b) if an ideal a of $k$ is principal in $K$, then a is principal in $k$.

Proof. Denote by $B$ the group of elements $\beta$ of $k^{\times 1)}$ such that $(\beta)$ is an $l$-th power of some ideal in $k$, and denote by $(5$ the group of ideal classes of $k$. Let $W$ be the group generated by $H$ and all $l$-th powers of elements of $k^{\times}$, and let

$$
B=B_{0} \supset B_{1} \supset \ldots \supset B_{s-1} \supset B_{s}=W
$$

be a sequence of subgroups of $B$ such that $\left(B_{i-1}: B_{i}\right)=l$ for every $i(1 \leqq i \leqq s)$. As preliminaries, we shall prove that, for every $i$, there is a prime ideal $\mathfrak{p}_{i}$ of $k$ which satisfies the following conditions: i) an element $\gamma$ of $B_{i-1}$ is an $l$ th power of some element in the $\mathfrak{p}_{i}$-adic field $k_{\mathfrak{p}_{i}}$ if and only if $\gamma$ belongs to $B_{i}$. ii) The set of ideal classes of $\mathfrak{p}_{1}, \ldots, \mathfrak{p}_{s}$ contains an independent base of $\left(5 / \mathbb{G}^{l}\right.$. Assume first that $k \neq \zeta_{l}$. Set $k_{l}=k\left(\zeta_{l}\right)$. Let $\Lambda=k_{l}(\sqrt[l]{\sqrt{B}})$ be the field obtained from $k_{l}$ by adjoining all $l$-th roots of elements of $B$. Then $A$ contains no cyclic extention of degree $l$ over $k$. For, if $L / k$ is cyclic of degree $l$, and $L \subset A$, then $k_{l} L / k$ is abelian, $k_{l} L / k_{l}$ is cyclic of degree $l$ and therefore $k_{l} L=k_{l}(\sqrt[l]{\beta})$, where

Received March 3, 1955.

1) We shall use this notation to stand for the multiplicative group of non-zero elements of a field. 
$\beta$ is an element of $B$. But this is impossible because $k_{l}(\sqrt[l]{\beta}) / k$ is apparently non-abelian. Thus we see that, if $Z_{l}$ is the class field over $\left(\varsigma^{l}\right.$, then

$$
k_{l}(\sqrt[l]{\wedge}) \cap Z_{l}=k .
$$

Let $\beta_{i}(1 \leqq i \leqq s)$ be an element of $B_{i-1}$ which does not belong to $B_{i}$. Set $k_{l}\left(\sqrt[l]{\beta_{i}}\right)=k_{i}, k_{l}\left(\sqrt[l]{B_{i}}\right)=k_{i}^{\prime}$. Then since $W$ contains all $l$-th powers of elements of $k^{\times}$and since every element of $k$, being an $l$-th power of some element in $k_{l}$, is already an $l$-th power of some element in $k^{2)}$ we have $k_{i} \cap k_{i}^{\prime}=k_{l}$. Therefore it gives infinitely many prime ideals $\mathfrak{q}_{i}$ of $k_{l}$ which are of degree 1 over $k$ and such that

$$
\left(\frac{k_{i} / k_{l}}{\mathfrak{q}_{i}}\right) \neq 1, \quad\left(\frac{k_{i}^{\prime} / k_{l}}{\mathfrak{q}_{i}}\right)=1 .
$$

Let $\widetilde{\mho}_{i}$ be the set of prime ideals $\mathfrak{p}_{i}$ of $k$ divisible by some $\mathfrak{q}_{i}$. Then since $k_{p_{i}}$ $=k_{l, \mathfrak{q}_{i}}$, the condition $\mathrm{i}$ ) is an immediate consequence of (3) and the theory of Kummer extentions. On the other hand, it is easily seen that $\widetilde{F}_{i}$ contains a prime ideal clase $^{3)}$ of $k$ with respect to $A$. To prove the condition ii), it is sufficient to show that every class of ideals of $k$ modulo $\left(s^{l}\right.$ contains a prime ideal of $\tilde{F}_{i}$. But this is actually the case because it follows from (2) that every prime ideal class of $k$ with respect to $A$ intersets with every class of ideals modulo ${ }^{l}{ }^{l}$. Now, assume that $k \ni \zeta_{l}$. Then every cyclic subfield over $k$ of $Z_{l}$ is of the form $k(\sqrt[l]{\beta})$, where $\beta \in B$. But the assumption in the theorem implies $\beta \notin W$. Therefore the elements $\beta_{1}, \ldots, \beta_{s}\left(\beta_{i} \in B_{i-1}, \notin B_{i}\right)$ can be so chosen that we have $Z_{l} \subset k\left(\sqrt[l]{\beta_{1}}, \ldots, \sqrt[l]{\beta_{s}}\right)$. Set, as before, $k_{i}=k\left(\sqrt[l]{\beta_{i}}\right), k_{i}^{\prime}$ $=k\left(\sqrt[l]{B_{i}}\right)$. Then our assertion follows immediately whenever we take $\mathfrak{p}_{i}$ with $\left(\frac{k_{i} / k}{\mathfrak{p}_{i}}\right) \neq 1,\left(\frac{k_{i}^{\prime} / k}{\mathfrak{p}_{i}}\right)=1$.

Making use of the condition $\mathrm{i}$ ), we can conclude that, for every $i(1 \leqq i \leqq s)$, there is a character $\chi_{i}$ of $k_{\mathfrak{p} i}^{\times}$which is of order $l$ and such that

$$
\chi_{i}\left(\beta_{i}\right) \neq 1, \quad \chi_{i}\left(\beta_{i}\right)=1 .
$$

Now, it follows from Grunwald's theorem that there are infinitely many cyclic extention $K / k$ of degree $l$ with following properties: I) Besides the prime ideals $\mathfrak{p}_{i}$, it gives one and only one prime ideal and no infinite place of

2) See Hasse [3], §1, Satz 1.

3) See Hasse [2], II, § 24 . 
$k$ which ramifies in $K^{4)}$ ii) There is an isomorphism $\varphi$ between the Galois group of $K / k$ and the group of all $l$-th roots of unity such that

$$
\left(\frac{\alpha, K / k}{\mathfrak{p}_{i}}\right)^{p}=\varkappa_{i}(\alpha)
$$

where $\alpha$ is an arbitrary element of $k^{\times}$. We propose to prove that the field $K$ has the required properties.

Let $a$ be an ideal of $k$. Assume that $\mathfrak{a}=(A)$, where $A \in K$. Then we have $\mathfrak{a}^{l}=N_{K / k} \mathfrak{a}=\left(N_{K / k} A\right)$. On the other hand, it follows from (4), (5) that $N_{K / k} A$ $\in W$. This means that $\left(N_{K / k} A\right)=(\alpha)^{l}$ for an element $\alpha$ of $k$, whence $\mathfrak{a}=(\alpha)$ and the property $b)$ is verified. To prove a), we make the following observation. Since from (4) and (5) follows, as before, $H \supseteqq N_{K / k} E_{K}$, it suffices to prove that

$$
\left(E_{k}: N_{K / k} E_{K}\right) \leqq\left(E_{k}: H\right)
$$

Denote by $a$ the group of ideals of $k$, by $(\alpha)$ the group of principal ideals of $k$, by $\mathfrak{H}_{0}$ the group of ambiguous ideals of $K / k$ and by $\left(A_{0}\right)$ the group of principal, ambiguous ideals of $K / k$. Let further $E_{0}$ be the group of units $E_{0}$ of $K$ such that $N_{K / k} E_{0}=1$, and let $\sigma$ be a generator of the Galois group of $K / k$. Then we obtain easily the following relations:

$$
\begin{aligned}
& \left(\mathfrak{H}_{0}: \mathfrak{a}\right) /\left(\mathfrak{H}_{0}:\left(A_{0}\right) \mathfrak{a}\right)=\left(\left(A_{0}\right):(\alpha)\right) /\left(\left(A_{0}\right) \cap \mathfrak{a}:(\alpha)\right), \\
& \left(\mathfrak{H}_{0}: \mathfrak{a}\right)=l^{s+1}, \\
& \left(A_{0}\right) /(\alpha) \cong E_{0} / E_{K}^{1-\sigma} .
\end{aligned}
$$

Since the condition ii) is satisfied, we may assume that the set of ideal classes of $p_{1}, \ldots, p_{t}$ is an independent base of $\left(5 / \tilde{\Xi}^{l}\right.$, where $t$ is determined by $l^{t}$ $=\left(\mathfrak{s}:(\mathbb{l},)^{l}\right)$. Now assume that $\mathfrak{p}_{i}=\mathfrak{P}_{i}^{l}$ in $K$ and that $\mathfrak{P}_{1}^{\nu_{1}} \ldots \mathfrak{P}_{t}^{\nu^{t}} \in\left(A_{0}\right) a$. Then

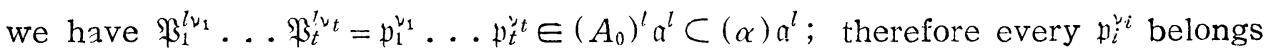
to an ideal class of $e^{l}$. Thus we have

$$
\left(\mathfrak{F}_{0}:\left(A_{0}\right) \mathfrak{a}\right) \geqslant l^{t} .
$$

Furthermore, the property b) implies

$$
\left(\left(A_{0}\right) \cap \mathfrak{a}:(\alpha)\right)=1
$$

4) See Hasse [3], "Starker Existenzsatz (zykilscher Fall mit Primzahlpotenzordnung)" at p. 45, especially its "Genauer"-part. In the case of prime degree $l$, this theorem is applicable without any extention of the set $D$, as we learn from its proof. 
and finally we can conclude by means of Herbrand's lemma ${ }^{5)}$ that

$$
\left(E_{0}: E_{K}^{1-\sigma}\right)=l\left(E_{k}: N_{K / k} E_{K}\right) .
$$

It follows from (7), (8), (9), (10), (11) and (12) that $l^{s+1} / l^{t} \geqslant l\left(E_{k}: N_{K / k} E_{K}\right)$, whence $\left(E_{k}: N_{K / k} E_{K}\right) \leqq l^{s-t}$, which shows that (6) is true. The theorem is thereby completely proved.

Corollary. $k$ and $E_{k}$ being the same as in the theorem, let $l$ be a prime number which does not divide either the class number of $k$ or the number of roots of unity in $k$, and let $H$ be any subgroup of $E_{k}$ containing all l-th powers of elements of $E_{k}$. Then there are infinitely many cyclic extentions $K / k$ of degree $l$ with the properties $a$ ) and $b$ ).

\section{REFERENCES}

[1] Chevalley, C., Class field theory, Nagoya University (1953/54).

[2] Hasse, H., Bericht, I (1926), I $a$ (1927) and II (1930).

[3] Hasse, H., Zum Existenzsatz von Grunwald in der Klassenkörpertheorie, J. Reine Angew. Math., 188 (1950), 40-64.

[4] Whaples, G., Non-analytic class field theory and Grunwald's theorem, Duke Math. J., vol. 9 (1942), 455-473.

Mathematical Institute, Nagoya University

5) See Chevalley [1], $\$ 10$. 